\title{
ENGINEERING, DESIGN AND PROTOTYPE TESTS OF A 3.9 GHZ TRANSVERSE-MODE SUPERCONDUCTING CAVITY FOR A RADIOFREQUENCY-SEPARATED KAON BEAM
}

\author{
Mark Champion, Leo Bellantoni, Tim Berenc, Craig Deibele, Helen Edwards, Mike Foley, \\ Joel Fuerst, Moyses Kuchnir, Allan Rowe, FNAL*, Batavia, IL 60510, USA
}

\begin{abstract}
A research and development program is underway to construct superconducting cavities to be used for radiofrequency separation of a Kaon beam at Fermilab. The design calls for installation of twelve 13-cell cavities operating in the $3.9 \mathrm{GHz}$ transverse mode with a deflection gradient of $5 \mathrm{MV} / \mathrm{m}$. We present the mechanical, cryogenic and vacuum design of the cavity, cryomodule, rf power coupler, cold tuner and supporting hardware. The electromagnetic design of the cavity is presented in a companion paper by Wanzenberg and McAshan [1]. The warm tuning system (for field flatness) and the vertical test system will be presented along with test results of bench measurements and cold tests on single-cell and five-cell prototypes.
\end{abstract}

\section{INTRODUCTION}

The CKM (Charged Kaons at the Main injector) experiment at Fermilab proposes to use the technique of $\mathrm{rf}$ separation to produce a charged kaon beam from a mixed beam of protons, pions and kaons. Two deflecting stations are planned, each consisting of six $3.9 \mathrm{GHz}$ superconducting Niobium cavities operating at a deflection gradient of $5 \mathrm{MV} / \mathrm{m}$. The Main Injector has a cycle time of three seconds with a beam spill of one second, hence the choice to use superconducting cavities. The cavity frequency and gradient were conservatively chosen based on the state of the art in superconducting rf cavities. A frequency of $3.9 \mathrm{GHz}$ and a kaon beam energy of $22 \mathrm{GeV} / \mathrm{c}$ results in a distance of 86 meters between the deflection stations. Further information on the layout and design considerations of the deflection system are presented in the design report [2].

\section{CAVITY DESIGN, FABRICATION AND TUNING}

\subsection{Cavity Design}

The electromagnetic design of the cavity is presented in detail by McAshan and Wanzenberg [3]. They compare several designs having different curvatures at the iris and equator regions and having different iris and equator radii. The design was optimized based on consideration of shunt impedance, maximum surface magnetic fields, and the spacing between the $\pi$ mode and the $\pi$-1 mode. The iris diameter is $30 \mathrm{~mm}$ whereas the beam tube diameter is $36 \mathrm{~mm}$. The end cells have a slightly different shape to

\footnotetext{
* Fermilab is operated by the Universities Research Association under contract to the U.S. Department of Energy.
}

compensate for end effects. Four ports are located on the beam tubes: one for the rf power coupler, one for a pickup probe, and two for higher order mode couplers, which are not expected to be necessary. The cavity design is shown in Figure 1.

\subsection{Cavity Fabrication}

Three prototype Niobium cavities have been fabricated to date: one five-cell of design A15 and two single-cells of design C15. The five-cell cavity was fabricated from reactor grade $\mathrm{Nb}$ to verify drawing, machining and electron beam welding techniques. The single-cell cavities were fabricated from high quality $\mathrm{Nb}$ (RRR 300). The cavity half cells are drawn from $1.6 \mathrm{~mm}$ plates. The iris welds are performed in two passes, one exterior and one interior, to form dumbbells. These are joined to each other via exterior equator welds. The beam tubes are rolled and seam welded and $16 \mathrm{~mm}$ diameter pull-outs are made for the side ports. The cavity flanges, including beam tube, side ports and helium tank, are fabricated from $\mathrm{Nb}$-Ti. The titanium helium tank is demountable. The beam tube and side port flange joints use solid aluminum seals of hexagonal cross section. One of the single-cell cavities has the complete end package including side ports and $\mathrm{He}$ tank flanges and is shown in Figure 2.

The production of the prototype cavities has been a collaborative effort. The dies are produced by Rutgers University, the machining and drawing is performed in the Fermilab machine shops, and the electron beam welding is performed at Sciaky, Inc. Prior to drawing, the half-cell plates are scanned for impurities at DESY using eddy current techniques. Buffered chemical polishing (BCP) of complete cavities has been performed at Jefferson Lab.

Future prototype production calls for two one-cell cavities, two three-cell cavities, and two 13-cell cavities. The production cavities will be procured from industry.

\subsection{Cavity Tuning}

The dies used to form the half cells are unpolarized, i.e., they are azimuthally symmetric. Polarization is achieved after the cavity is fully assembled by squeezing each cell in a press to produce a deformation of about $4 \mathrm{~mm}$ on the diameter. This aligns the two polarizations of the $\pi$ mode along the length of the cavity and splits them by about $20 \mathrm{MHz}$. Subsequently the cells of the cavity are stretched or compressed as necessary to achieve field flatness and the correct frequency for the $\pi$-mode. We have chosen to operate on the lower frequency polarization, which happens to be the lowest frequency mode of the passband. 


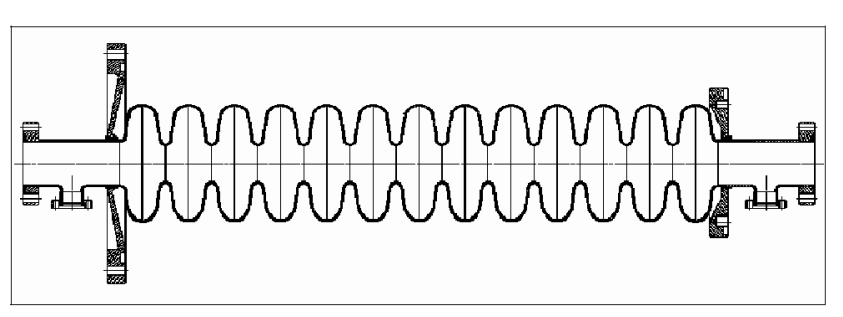

Figure 1. Cavity profile including complete end package.

The five-cell prototype cavity was tuned using a relatively simple tuner that provides for stretching or compressing each cell. The deflection is measured by means of dial indicators. The alignment of the tuner axis relative to the cavity axis is somewhat uncertain due to the simplicity of the tuner, hence some mode rotation was observed during the tuning process. A more sophisticated tuner has been designed and is presently under construction. It will be used for tuning the first 13-cell prototype cavity later this year.

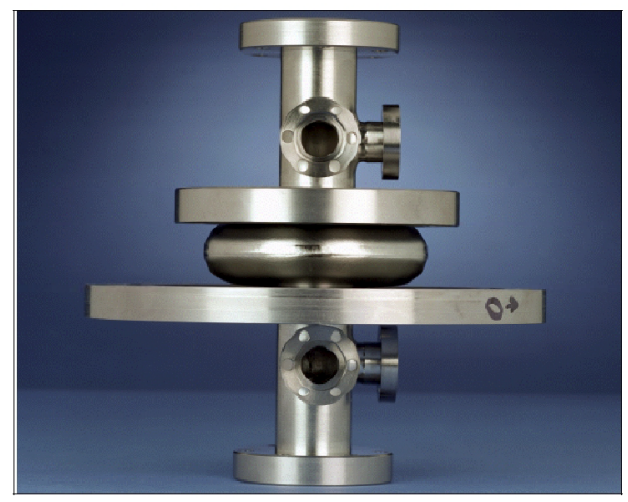

Figure 2. Single-cell cavity with complete end package.

Beadpull measurements have been performed to measure the field flatness and the orientation of the deflecting mode relative to the polarization flats. Spherical and needle shaped beads have been used. These measurements are described in detail in a tuning note by Bellantoni [4].

\section{CRYOMODULE DESIGN}

\subsection{Cryomodule Layout}

The cryomodule design calls for two cavities per module as depicted in Figure 3. The helium tanks are rigidly connected at the center to form a two-cavity unit that will be suspended from hangers at each end. The design calls for a vacuum vessel, $80 \mathrm{~K}$ thermal shield, magnetic shielding, and the cavity/helium vessel at $1.8 \mathrm{~K}$. The central tower forms the cryogenic feedcan for the cryomodule.

\subsection{Cold Tuner}

A cold tuning system similar to the TESLA cold tuner is under development. The tuning system will include levers, living hinges, ball bearing pivots, a gearbox and a stepping motor, all operating within the insulating vacuum of the cryomodule.

\subsection{RF Coupler}

Each cavity will have an input rf power coupler and a weakly coupled pickup probe for monitoring the cavity fields. The power couplers will mount vertically from beneath the cavity at the center of the cryomodule whereas the pickup probes will be at the ends of the cryomodule. The external Q of the power coupler is approximately 6e7 resulting in a loaded bandwidth of $65 \mathrm{~Hz}$. The power coupler will be designed for adjustable coupling over one order of magnitude and will handle up to $400 \mathrm{~W}$ continuous wave.

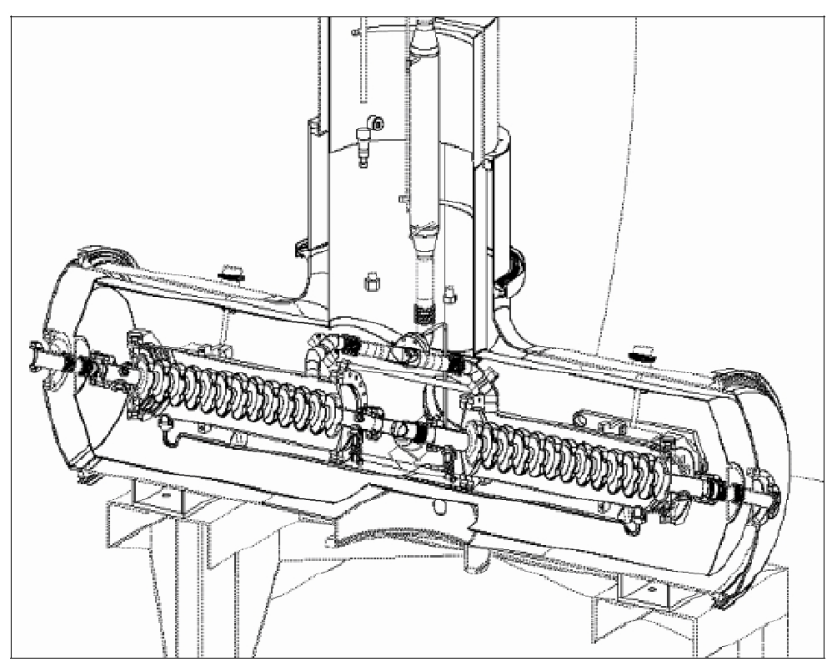

Figure 3. The two-cavity cryomodule.

\section{VERTICAL DEWAR TEST RESULTS}

\subsection{Five Cell Cavity}

The first tests were performed on the five-cell prototype. The cavity was BCP etched at Jefferson Lab and low pressure rinsed with ultrapure water prior to testing. The uniformity of surface removal was suspect due to the unavoidable high flow rate during etching. The resulting Qo versus deflecting gradient curve is shown in Figure 4. The cavity had a lower than expected Qo and quenched at less than $1 \mathrm{MV} / \mathrm{m}$ deflecting gradient. It exhibited Q switching behavior at $4.5 \mathrm{~K}$, but not at $1.8 \mathrm{~K}$. A cooldown measurement of Qo as a function of temperature from $4.5 \mathrm{~K}$ to $1.5 \mathrm{~K}$ was performed and the results are shown in Figure 5 in the form of Rs versus $\mathrm{Tc} / \mathrm{T}$, where $\mathrm{Tc}=9.2 \mathrm{~K}$ is the critical temperature for Niobium. The residual resistance of $340 \mathrm{n} \Omega$ is inexplicably high. Recently the cavity has been retested after further BCP etching and high pressure rinsing. The cavity Qo improved to about $2 \mathrm{e} 9$ and the maximum deflecting gradient reached before quenching was about $1.5 \mathrm{MV} / \mathrm{m}$. Unlike during the first test, the Q switch behavior was observed at $1.8 \mathrm{~K}$ as shown in Figure 4. 


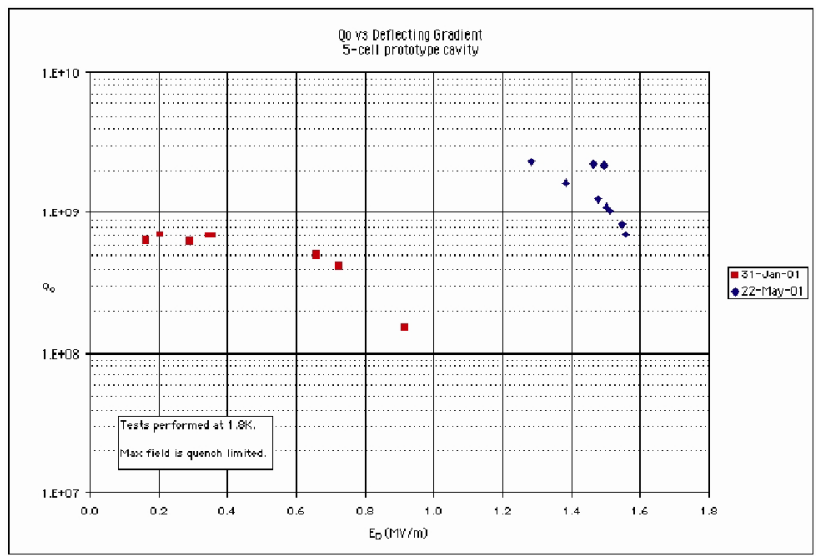

Figure 4. Curves of Qo versus deflecting gradient for the five-cell prototype.

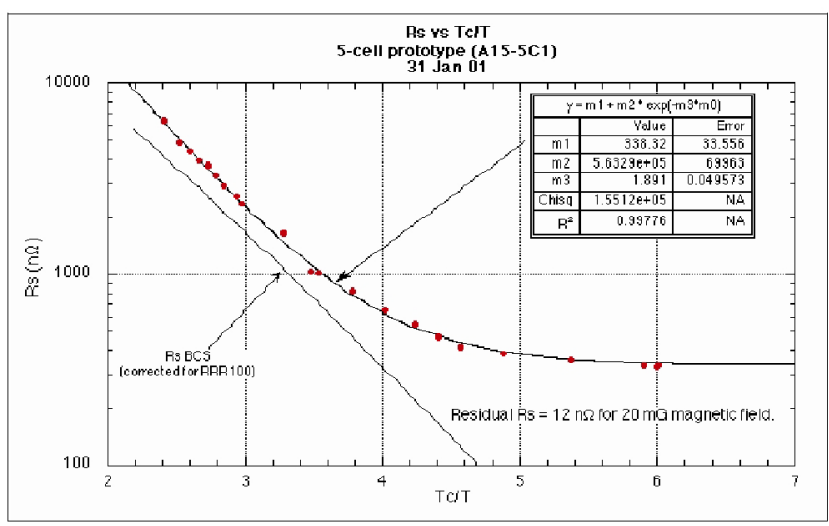

Figure 5. Curve of surface resistance Rs versus inverse temperature for the five-cell prototype.

\subsection{Single Cell Cavity}

The first single-cell cavity was BCP etched and high pressure rinsed at Jefferson Lab prior to testing. The results of the Qo versus deflecting gradient measurement are shown in Figure 6. The cavity quenched at about $9.5 \mathrm{MV} / \mathrm{m}$. X-ray production indicated electron field emission due to particulate contamination. The assembly of the cavity to the test stand was problematic and required three attempts before a vacuum tight connection could be made, so the field emission was not a surprise.

At the same time a high pressure water rinsing system was being commissioned at Fermilab. Therefore the cavity was dismounted, rinsed, and remounted for a second test. Measurements taken on 26-27 April and show a marked improvement in the cavity performance. No field emission was observed and there was a small improvement in the maximum attainable deflecting gradient prior to quenching.

The results of a cooldown Qo versus temperature measurement are presented in Figure 7. The agreement with the predicted BCS surface resistance, Rs, is quite good, and a residual resistance of about $60 \mathrm{n} \Omega$ was achieved.

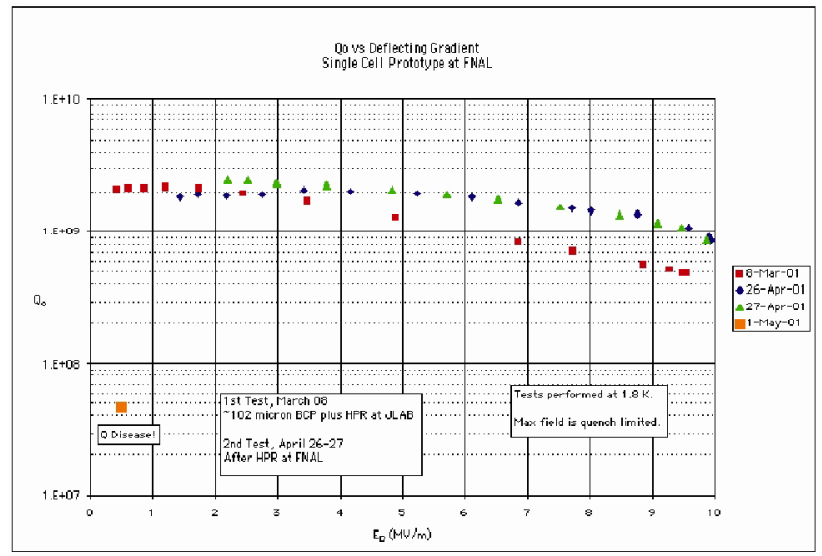

Figure 6. Curves of Qo versus deflecting gradient for the single-cell prototype.

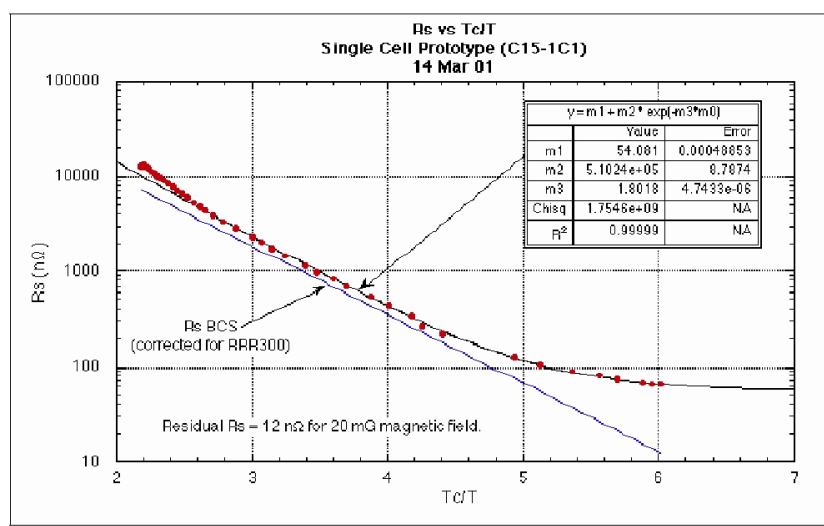

Figure 7. Curve of surface resistance Rs versus inverse temperature for the single-cell prototype.

\section{CONCLUSION}

Prototype superconducting rf separator cavities have been fabricated and tested at Fermilab in support of the proposed CKM experiment. A single-cell cavity fabricated from RRR300 Niobium has exceeded the performance goals of the project. The achieved deflecting gradient of $10 \mathrm{MV} / \mathrm{m}$ corresponds to peak electric and magnetic fields of $33.6 \mathrm{MV} / \mathrm{m}$ and $109 \mathrm{mT}$, respectively. Scaled to a 13-cell cavity, this implies a deflecting gradient of $7.1 \mathrm{MV} / \mathrm{m}$.

\section{REFERENCES}

[1] R. Wanzenberg et al, "Design and Measurements of a Deflecting Mode Cavity for an RF Separator," Proceedings of the 2001 Particle Accelerator Conference, Chicago, 2001.

[2] D. A. Edwards, Editor, "An RF Separated Kaon Beam from the Main Injector: Superconducting Aspects," Fermilab TM-2060, Batavia, 1998.

[3] M. McAshan and R. Wanzenberg, "RF Design of a Transverse Mode Cavity for Kaon Separation," Fermilab TM-2144, Batavia, 2001.

[4] Leo Bellantoni, "Notes on Tuning the 5-cell Cavity", Fermilab CKM Note 34, Batavia, 2000. 